Convergencia tecnológica y concentración mediática. Un apunte sobre la fusión Cablevisión-Telecom desde la economía política de la comunicación y la cultura

Serguei Komissarov, Emiliano Sánchez Narvarte

Tram[p]as de la comunicación y la cultura (N. ${ }^{\circ} 83$ ), e029, abril-septiembre 2018

ISSN 2314-274X | https://doi.org/10.24215/2314274Xe029

http://perio.unlp.edu.ar/ojs/index.php/trampas

FPyCS | Universidad Nacional de La Plata

La Plata | Buenos Aires | Argentina

\title{
CONVERGENCIA TECNOLÓGICA Y CONCENTRACIÓN MEDIÁTICA
}

UN APUNTE SOBRE LA FUSIÓN CABLEVISIÓN-TELECOM DESDE LA ECONOMÍA POLÍTICA DE LA COMUNICACIÓN Y LA CULTURA

TECHNOLOGICAL CONVERGENCE AND MEDIA CONCENTRATION

A NOTE ON THE CABLEVISIÓN-TELECOM MERGER FROM

THE POLITICAL ECONOMY OF COMMUNICATION AND CULTURE

Serguei Komissarov Emiliano Sánchez Narvarte

skomissarov@protonmail.com emiliano.sanchez@perio.unlp.edu.ar

https://orcid.org/0000-0002-7785-8369 https://orcid.org/0000-0002-5407-3681

\section{Instituto de Estudios Comunicacionales en Medios, Cultura y Poder Aníbal Ford Facultad de Periodismo y Comunicación Social Universidad Nacional de La Plata | Argentina}

\begin{abstract}
Resumen
Abstract

Los autores analizan la relación entre la The authoress analyzes the relationship convergencia tecnológica y la concentración mediática, a partir de la fusión entre Cablevisión y Telecom, para lo cual se describe el estado actual del mercado argentino, las políticas de comunicación y su incidencia sobre las actividades de las industrias del sector. between technological convergence and media concentration, from the merger between Cablevisión and Telecom, for wich the current state of the Argentine market, communication policies and their impact on the activities of the industries in the sector are described.
\end{abstract}

Palabras clave | convergencia, concentración, políticas, comunicación Keywords | convergence, concentration, policies, communication 


\title{
CONVERGENCIA TECNOLÓGICA Y CONCENTRACIÓN MEDIÁTICA
}

UN APUNTE SOBRE LA FUSIÓN CABLEVISIÓN-TELECOM DESDE LA ECONOMÍA POLÍTICA DE LA COMUNICACIÓN Y LA CULTURA

\author{
Por Serguei Komissarov
}

y Emiliano Sánchez Narvarte

\section{Introducción}

A fines de junio de 2017 se anunció la fusión entre Cablevisión y Telecom. La operación genera las condiciones para conformar el mayor conglomerado infocomunicacional del mercado argentino a un nivel inédito, incluso, para el resto de América Latina (Sel, 2010; Dantas, 2013; Becerra \& Mastrini, 2017). De esta manera, Cablevisión-Telecom concentrará a nivel nacional 39\% de la telefonía fija, 33\% de la telefonía móvil, 55\% de las conexiones a Internet de banda ancha y 39\% de la TV paga (Iglesias, 2017).

Basta decir que de acuerdo a los balances presentados ante la Comisión Nacional de Valores (CNV) y la Bolsa de Comercio de Buenos Aires, correspondientes a 2017, el Grupo Telecom informó utilidades por un total de $\$ 5.641$ millones, $128,3 \%$ superiores a los $\$ 2.471$ millones contabilizados en 2016. ${ }^{1}$ Cablevisión, por su parte, ganó \$ 4.556 millones, cifra que estuvo $40,4 \%$ por encima de los $\$ 3.244$ millones del año anterior. ${ }^{2}$

En términos de valor bursátil, el precio del conglomerado ascendería a 13 mil millones de dólares, lo que supera a las dos empresas de mayor valor en el país: Mercado Libre (12 mil millones de dólares) y la empresa petrolera del Estado, YPF (9.500 millones de dólares).

En diciembre de 2017, el Ente Nacional de Comunicaciones (ENaCom), mediante la Resolución 5644, autorizó la fusión sujeta al cumplimiento de ciertos requerimientos pretendidamente antimonopólicos. 
Actualmente, la Comisión Nacional de Defensa de la Competencia, dependiente de la Secretaría de Comercio del Ministerio de Producción, es el órgano oficial que tiene la última palabra para permitir la fusión. Por su parte, distintas organizaciones de la sociedad civil denunciaron que con la resolución del ENaCom se violan la Ley de Defensa de la Competencia (25.156/99) y la Ley de Protección y Promoción de la Diversidad de las Expresiones Culturales (26.305/07), y solicitaron la nulidad de la medida.

De forma contraria a lo que parecía ser un proceso sin mayores complicaciones, las tensiones políticas, jurídicas y económicas emergentes abren interrogantes sobre la resolución del caso.

A lo largo de este artículo, nos proponemos:

- Analizar algunas consideraciones teórico metodológicas para pensar la relación entre la convergencia tecnológica y la concentración mediática.

- Realizar una breve descripción del estado actual del mercado infocomunicacional argentino.

- Relevar las políticas de comunicación del período 2015-2017 y analizar su incidencia sobre las actividades de las industrias del sector.

- Describir y analizar la fusión entre Cablevisión y Telecom, y sus efectos sobre la concentración del mercado.

- Establecer una serie de interrogantes sobre el modo en el que la reestructuración del mercado incide sobre el derecho a la libertad de expresión, la circulación de información y el acceso a la cultura.

\section{Consideraciones teórico metodológicas}

Para pensar el proceso de articulación entre la convergencia tecnológica y la concentración de las industrias infocomunicacionales, ${ }^{3}$ utilizaremos el enfoque de la economía política de la comunicación y la cultura (EPCC) cuyo objeto de estudio son "las relaciones sociales (particularmente, las relaciones de poder) que constituyen la producción, la distribución y el consumo de bienes 
simbólicos (Bolaño \& Mastrini, 2002). Su principal unidad de análisis son las industrias culturales, que deben ser consideradas desde un enfoque histórico estructural (Sánchez Ruiz, 1991) y circunscrito a la dinámica del capitalismo, entendido como un modo histórico de producción económica, por un lado, y como una forma de organización social, cultural y politica, por el otro, caracterizado por la dominación de un sistema abstracto y complejo de relaciones de cambio (Garnham, 1990). Desde esta perspectiva, se busca analizar cómo los sistemas comunicativos son socialmente construidos en el contexto del sistema capitalista (Mosco, 2009).

La economía política de la comunicación y la cultura intenta comprender cuáles son las condiciones en las cuales se instituyen las regulaciones politicas, económicas, sociales y tecnológicas (Williams, [1974] 1997) de la comunicación, reconociendo como objeto de estudio el doble carácter ideológico y económico de los bienes y los servicios de la cultura. No tomar en cuenta las condiciones y las regulaciones económicas, ni las circunstancias sociales en las que se produce y circula la comunicación genera el riesgo de elaborar un análisis centrado únicamente en las novedades y en los cambios tecnológicos. Del mismo modo, si solo se enfatiza en los cambios normativos, y se omiten sus efectos estructurales y sus modos de aplicación y de apropiación social, también se cae en un reduccionismo indeseado (Becerra, 2015).

Precisamente por eso, se recuperan las conceptualizaciones de Lawrence Lessig (1988), quien propone pensar la regulación de los espacios atravesados por las tecnologías digitales en cuatro dimensiones: las disposiciones legales de las políticas públicas de comunicación; la configuración de los mercados (que, a su vez, determinan el acceso a los bienes y a los servicios por parte de los distintos grupos sociales); las nuevas formas de producción, de circulación y de consumo que posibilita la convergencia tecnológica; y la morfología de cada sector informacional (con su arquitectura y sus códigos específicos).

Para analizar el marco regulatorio que rige las actividades de las empresas infocomunicacionales, se utilizarán algunas herramientas propias de la metodología en investigación jurídico-social (Kerlinger \& Lee, 2002). Esta perspectiva privilegia el funcionamiento o los efectos del derecho en una determinada sociedad en lugar de interesarse solamente por las normas en abstracto y por la relación que guardan entre ellas. 
Para estudiar la estructura y la morfología de los mercados infocomunicacionales se utilizará el Monitoreo de la Propiedad de Medios (MOM - Media Ownership Monitor). Esta metodología, desarrollada por el Center for Media Pluralism and Media Freedom (CMPF) del Instituto Universitario Europeo, evalúa el nivel de control en la propiedad de los medios de comunicación masiva y sus efectos sobre el pluralismo informativo mediante una serie de indicadores que abordan tres dimensiones de análisis: económica, jurídica y política.

Entre otros indicadores MOM, podemos mencionar la concentración de la propiedad horizontal en los respectivos sectores de los medios de comunicación (televisión, radio, prensa, Internet), tomando en cuenta la cuota de mercado (porcentaje de los ingresos totales), la concentración de la audiencia, la transparencia de propiedad y de los intereses afiliados, y la financiación de medios por la publicidad.

Otro indicador para el estudio de la concentración mediática será el Índice Hirschman-Herfindahl (IHH). Con este método se pretenden cuantificar las características de la estructura de la industria y la distribución del tamaño de las firmas (Miguel de Bustos, 1993). Estos tipos de indicadores son los comúnmente utilizados en las investigaciones y en los análisis sobre fusiones y competencia (Castañeda Véliz, 2007).

El mercado de las comunicaciones convergentes en la Argentina

El creciente avance tecnológico transforma las industrias culturales y permite disponer de una enorme cantidad de servicios destinados a la transmisión de contenidos multimedia. Estas innovaciones posibilitan nuevas formas de producción, de circulación y de consumo de bienes culturales, al tiempo que modifican el mercado de los medios audiovisuales y las telecomunicaciones.

Una de las características salientes de la digitalización de la información es la convergencia tecnológica (Jenkins, 2006; Miller, 2011; Meikle \& Young, 2011; Peil \& Sparviero, 2017). Por un lado, la creciente estandarización del formato digital facilita la grabación, el almacenamiento y la transmisión de la información. Por el otro, permite la integración de las distintas redes de datos 
para transportar servicios y señales similares. De esa manera, el formato digital — sea voz, sonido, texto o audiovisual- no se restringe a un único medio y permite recibir diversos servicios a través de un mismo dispositivo.

En la práctica, la convergencia tecnológica permite brindar: telefonía fija, comunicaciones móviles, acceso a Internet - tanto fija como móvil- y televisión por suscripción, ya sea por medio físico (cable) o inalámbrico (satelital). Este conjunto de servicios es ofrecido por un mismo operador, mediante una misma red y se cobra como un paquete, generalmente, denominado cuádruple play.

\section{Telefonía fija y acceso a Internet}

En 1990 se llevó a cabo la privatización de la Empresa Nacional de Telecomunicaciones (ENTEL), fundada el 13 de enero de 1956. A partir de entonces, dos operadores telefónicos se dividieron el país: Telecom Argentina, el norte, y Telefónica Argentina, el sur. Cada uno mantuvo el monopolio en su zona de influencia hasta 1999. Ambas empresas estaban imposibilitadas de brindar servicios de telefonía móvil, datos, Internet o llamadas internacionales. Ese mercado quedó en manos de compañías como Movicom, Miniphone, Startel, Advance, Arnet, Telintar e Impsat.

Luego de la privatización, se digitalizó la totalidad de las centrales telefónicas, lo que permitió ofrecer servicios como transferencia de llamada, llamada en conferencia, discado directo entrante y el acceso al discado directo internacional.

A partir de 2000 comenzó una segunda digitalización de la red telefónica, que permitió brindar servicios de acceso a internet mediante banda ancha. A la línea digital del abonado, llamada Red Telefónica Conmutada (Public Switched Telephone Network), se le sumó una linea de suscriptor digital asimétrica o ADSL (Asymmetric Digital Subscriber Line). De esta manera, un mismo cable de cobre pasaba a transmitir dos señales en diferentes frecuencias: las bajas (de 0 a $4 \mathrm{Khz}$ ) para la telefonía fija, y las más altas (por encima de 28.5khz) para el ADSL. Con un filtro divisor y un módem, instalado en el domicilio del abonado, era posible acceder a internet mediante banda ancha, mientras que la línea telefónica quedaba libre para realizar o para recibir llamadas. 
Paralelamente, las empresas de televisión por cable comenzaron a brindar servicio de banda ancha, modificando sus redes con la tecnologia HFC (Híbrido de Fibra-Coaxial), para proveer Internet por cablemódem. A partir de entonces, los cableoperadores se convirtieron en la competencia directa de las empresas telefónicas en el ofrecimiento de este servicio.

De acuerdo al informe "Indicadores de Mercado TIC y Audiovisual», publicado por el ENaCom en diciembre de 2017, en el país existen 9.81 millones de líneas fijas frente a 62.23 millones de líneas móviles. La tendencia indica que el uso del teléfono fijo decae. Sin embargo, los clientes optan por mantener las líneas, lo que se explica por el hecho de que las redes de telefonía fija son utilizadas para el acceso a Internet mediante ADSL, mientras que el servicio de llamada pierde terreno ante la telefonía celular.

La competencia en el segmento de la telefonía fija entre Telefónica y Telecom es casi inexistente. Hay un oligopolio muy marcado: Telefónica posee $45 \%$ del mercado, mientras que Telecom llega a 39\%. El resto se reparte entre operadores menores. En telefonía celular, por el contrario, ambas compañias se disputan el servicio bajo sus respectivas marcas: Movistar y Personal. Lo mismo sucede con el acceso a Internet mediante ADSL. Telefónica ofrece su proveedor, Speedy, mientras que Personal hace lo propio con Arnet.

En 2017, el acceso a internet fija sumó un total de 7,72 millones de abonados, con un sostenido aumento desde 2008 (ENaCom, 2017). Si esta cifra se segmenta por la tecnología que utiliza el prestador del servicio, se observa que las empresas telefónicas (ADSL) y los cableoperadores (cablemódem) suman, entre sí, $94,4 \%$ del mercado: $47,51 \%$ y 46,87\%, respectivamente. Si se considera la totalidad del mercado, Cablevisión-Fibertel (Grupo Clarín) tiene 31\%; Telefónica-Speedy (España), 26\%; y Telecom-Arnet (Fintech y otros), 24\%.

\section{Telefonía móvil}

En 1989, la empresa Movicom comenzó a brindar el servicio de telefonía móvil en el Área Metropolitana de Buenos Aires (AMBA). En 1993, con la digitalización mediante la incorporación de la tecnología $2 \mathrm{G}$, fue posible mejorar la velocidad del tráfico de datos, además de integrar en una misma 
señal, junto con la voz, otros servicios como los mensajes de texto. El mismo año, la empresa Miniphone inició el servicio de 2G en el AMBA, mientras que, en 1995, CTI hizo lo propio en el interior del país.

Con la reducción del tamaño, el peso y el precio de los equipos, en 1996, Telecom Personal y Telefónica entraron también en el mercado de las telecomunicaciones móviles. En 1998, Nextel Argentina ofreció, en principio, el servicio de llamadas grupales denominado PTT (Push to Talk) y, más tarde, integró la telefonía tradicional junto al SMS.

En 1999, el Estado otorgó las licencias para explotar las bandas de $1900 \mathrm{MHz}$. Esto posibilitó que los operadores del interior del país pudieran expandir su cobertura a la zona del AMBA. A su vez, Movicom y Nextel pudieron comenzar a brindar servicios en todo el país. Miniphone, por su parte, se escindió de sus empresas controlantes (TECO y TASA) y se integró por mitades a Telecom Personal y a Unifon, nuevo nombre que adoptó Telefónica Comunicaciones Personales, perteneciente a Telefónica Argentina. En 2005, Telefónica adquirió Movicom y la operadora del grupo pasó a llamarse Telefónica Móviles de Argentina y a ofrecer sus servicios bajo la marca Movistar.

En 2001 comenzó a implementarse la tecnología GSM, que permitía conectarse a través del teléfono celular a una computadora, enviar y recibir mensajes por correo electrónico, fax, navegar por Internet y utilizar otras funciones digitales de transmisión de datos, como el servicio de mensajes de texto. Para 2006, todos los operadores del país adoptaron este sistema.

En 2007, Claro (CTI), Personal y Movistar, comenzaron a brindar el servicio 3G. Esta tecnología permitió el acceso a Internet desde el celular e inauguró la banda ancha móvil, capaz de transferir todo tipo de datos, como imágenes, voz y video. La irrupción en el mercado de los teléfonos inteligentes (smartphones) provocó que el tráfico de datos superara ampliamente el tráfico de voz. En tanto, el creciente parque de estos equipos terminó saturando la red móvil 3G, al exigir más ancho de banda debido a la intensificación del volumen de información por el intercambio de fotos, de videos y de música. 
Para resolver el incremento en la demanda, en 2014, el Estado Nacional licitó diez lotes de frecuencias de 3G y 4G LTE en las bandas de 700, 1700 y 2100 $\mathrm{MHz}$. Esta tecnología posibilita una mayor velocidad de conexión y un uso más eficiente del espectro comparado con el 3G. Las nuevas frecuencias ampliaron el ancho de banda en $214 \mathrm{MHz}$ (34 MHz destinados a 3G y $180 \mathrm{MHz}$ para 4G), lo que aumentó de 170 a $384 \mathrm{MHz}$ el espectro radioeléctrico dedicado exclusivamente a la telefonía móvil.

El monto recaudado por el Estado Nacional fue de 18.000 millones de pesos. Las empresas beneficiadas en la licitación fueron: Telefónica Móviles Argentina (Movistar), Telecom (Personal), AMX Argentina (Claro) y Arlink (Grupo Manzano-Vila). En Telefonía móvil, el mercado también se divide en tres: Claro (Carlos Slim) posee el 31\%, Telefónica (España) 33\% y Telecom (Fintech y otros) $31 \%$. Allí también participa Clarín con Nextel, dueño apenas de $2 \%$, pero con aspiraciones a competir con servicios de $3 \mathrm{G}$ y $4 \mathrm{G}$.

Personal comenzó a brindar el servicio 4G en diciembre del 2014 en Buenos Aires, Córdoba, Rosario, Mar del Plata, Pinamar, Salta y Santa Fe. También se comprometió a cubrir, en 18 meses, las ciudades más importantes del país, tal como lo exige la primera etapa de la licitación. En ese sentido, anunció invertir 1.400 millones de dólares en un plazo de tres años para la ampliación de servicios $3 \mathrm{G}$ y $4 \mathrm{G}$, sumados a los 411 millones de dólares que ya pagó por las frecuencias licitadas. A principios de 2018, Telecom - ahora bajo el control del grupo Clarín tras la fusión con Cablevisión- prometió una inversión trianual por U\$S 5.000 millones.

Movistar también inició su servicio 4G a fines de 2014 en el AMBA, Mar del Plata, Pinamar y Cariló. Durante 2015, anunció inversiones por 500 millones de dólares para mejorar las redes en Buenos Aires, Córdoba, Mendoza y Santa Fe. A esta cifra se le deben adicionar los 427 millones de dólares que pagó por la adjudicación de las nuevas licencias. Además, a principios de 2018, Telefónica anunció que planea invertir \$50.737 millones en el periodo 2018-2020.

Claro, en tanto, comenzó a brindar el servicio de 4G en junio de 2015. Posee redes en Buenos Aires, en Mendoza y en Córdoba, y espera completar la cobertura de todo el país en los próximos tres años. La empresa anunció que invertirá 2.650 millones de dólares durante cinco años, además de los 281 millones de dólares que ya desembolsó para la adquisición del nuevo espectro. ${ }^{4}$ 


\section{Televisión por suscripción}

En 1981 se fundó Cablevisión. Tan solo 15 años después, se conviertió en una de las más grandes operadoras de TV por cable de la Argentina. Parte de su éxito se debió a que, en 1997, comenzó a ofrecer Internet por cablemódem a través de Fibertel como una alternativa al servicio brindado por las telefónicas. Ese mismo año, Cablevisión y Multicanal compraron y se repartieron, en parte iguales, la empresa Video Cable Comunicación S.A. (VCC). Con esta operación, ambas compañias se transformaron en líderes del sector a nivel nacional detentando, cada una, el 25\% del mercado de la TV paga y el 15\% de los accesos a Internet.

En octubre de 2005, el Grupo Clarín y el fondo de inversión Fintech, a cargo del mexicano David Martínez, compraron cada uno el 25\% de Cablevisión. Por otro lado, Multicanal, que había logrado constituirse en una empresa importante dentro del mercado de la TV paga, fue adquirida por Clarín en 1991. Al año siguiente, Multicanal y Cablevisión anunciaron su fusión conformando una nueva sociedad. Cablevisión vendió su 40\% al Grupo Clarín y a Fintech, y fue absorbida por Multicanal, manteniendo su marca. A partir de entonces, Clarín pasó a controlar el $60 \%$ de la nueva empresa y Fintech el $40 \%$ restante. Esta proporción se mantuvo inalterada hasta 2017, cuando sobrevino la fusión Cablevisión-Telecom.

Esta reestructuración en la propiedad del multimedios posibilitó que, en 2006, Cablevisión concentrara 51\% del mercado de la TV paga.

En cuanto al servicio de acceso a Internet, la cuota de mercado de Fibertel $(11,6 \%)$, de Flash $(14,6 \%)$ y de Fullzero $(6,3 \%)$, como marcas de las compañías fusionadas, sumaban 32,5\%. Sus competidores directos eran Speedy (Telefónica) con 19\% y Arnet (Telecom) con 14\%.

Otra compañia que brinda el servicio de televisión paga es DirectTV, perteneciente a AT\&T, una empresa de telecomunicaciones estadounidense. DirectTV representa la competencia de todos los cableoperadores, ya que tiene una licencia que cubre todo el territorio nacional. Además, al transmitir su señal de manera inalámbrica mediante satélite, no está sujeta a invertir en tendido y puede cubrir la demanda donde no existe ningún tipo de red. Aunque en 2006 apenas contaba con 11\% del mercado, acaparaba 99,8\% de todos los abonados satelitales del país. 
El grupo nacional América (ex Grupo Uno), liderado por Daniel Vila y por José Luis Manzano, ofrece servicio de TV por cable, principalmente en la provincia de Mendoza y el oeste argentino, a través de su cable operador Supercanal. Telecentro, por su parte, cuenta con una red de TV por cable cuya extensión abarca Buenos Aires y el área metropolitana. Es la única competencia directa de Cablevisión en esa zona.

En tanto, se estima que existen unas 400 cooperativas telefónicas locales que operan en ciudades pequeñas en las que ni Telefónica ni Telecom poseen cobertura. Algunas de estas operadoras suministran televisión por cable y compiten, generalmente, con cooperativas eléctricas locales que ofrecen servicio de acceso a Internet o televisión paga. Finalmente, también existen unos 400 operadores PYME y varios cableoperadores cooperativos en poblaciones donde ni Cablevisión ni Supercanal se dedican a cubrir la demanda.

En 2017 se registró un total de 9,36 millones de accesos a la televisión paga (ENaCom, 2017). De ese total, 6,77 millones (72,3\%) corresponde a la TV por vínculo físico o radioeléctrico, y 2,53 millones (27\%) a la TV satelital. Como muestra la Tabla 1, en dicho mercado Cablevisión ronda el 40\% y DirectTV el $30 \%$. Los siguen Supercanal, con apenas 7\%, y Telecentro, con idéntica cifra.

\begin{tabular}{lccccc|}
\hline \multicolumn{1}{c}{ Empresa } & $\begin{array}{c}\text { Telefonía } \\
\text { fija }\end{array}$ & $\begin{array}{c}\text { Telefonía } \\
\text { móvil }\end{array}$ & $\begin{array}{c}\text { Banda } \\
\text { Ancha }\end{array}$ & TV paga & $\begin{array}{c}\text { TV } \\
\text { abierta }\end{array}$ \\
\hline Telefónica (España) & $45 \%$ & $\begin{array}{c}33 \% \\
\text { (Movistar) }\end{array}$ & $\begin{array}{c}26 \% \\
\text { (Speedy) }\end{array}$ & - & - \\
\hline Telecom (David Martínez) & $39 \%$ & $\begin{array}{c}31 \% \\
\text { (Personal) }\end{array}$ & $\begin{array}{c}24 \% \\
\text { (Arnet) }\end{array}$ & - & - \\
\hline Clarín & $0,2 \%$ & $\begin{array}{c}2 \% \\
\text { (Nextel) }\end{array}$ & $\begin{array}{c}31 \% \\
\text { (Fibertel) }\end{array}$ & $\begin{array}{c}\text { (Cablevisión) } \\
\text { (Artear) }\end{array}$ \\
\hline Claro (Telmex) & $3 \%$ & $34 \%$ & - & - & - \\
\hline Telefé (Viacom) & - & - & - & - & $33,6 \%$ \\
\hline Telecentro (Grupo Pierri) & $2 \%$ & - & $4 \%$ & $7 \%$ & - \\
\hline América (Grupo Vila Manzano) & - & - & $3 \%$ & $7 \%$ & $17 \%$ \\
\hline $\begin{array}{l}\text { Fecotel, Fecosur y Catel (Cooperativas } \\
\text { telefónicas/eléctricas) }\end{array}$ & $8 \%$ & $0,9 \%$ & $4,5 \%$ & $2 \%$ & - \\
\hline Red Intercable (Cables pyme) & $1 \%$ & - & $3,5 \%$ & $7 \%$ & - \\
\hline CCC Tucumán & - & - & - & $1 \%$ & - \\
\hline $\begin{array}{l}\text { Cablexpress/Gigared/Telered/Antina/ } \\
\text { Phonevision }\end{array}$ & $1 \%$ & - & $2 \%$ & $4 \%$ & - \\
\hline $\begin{array}{l}\text { Colsecor (Cables cooperativos y } \\
\text { pymes) }\end{array}$ & $0,4 \%$ & - & $1 \%$ & $3 \%$ & - \\
\hline $\begin{array}{l}\text { Albavisión (Canal 9) } \\
\text { DirecTV (AT\&T Time Warner) }\end{array}$ & - & - & - & - & $11,4 \%$ \\
\hline
\end{tabular}

Tabla 1 | Participación de todos los operadores en el mercado de comunicaciones convergentes Fuente: elaboración propia sobre la base de Iglesias (2017) 


\section{Politicas de comunicación y regulación convergente}

El 4 de enero de 2016, mediante el Decreto de Necesidad y Urgencia (DNU) 267, el Poder Ejecutivo Nacional creó el Ente Nacional de Comunicaciones (ENaCom) y modificó artículos centrales de la Ley de Servicios de Comunicación Audiovisual (LSCA 26.522/09) y de la Ley de Telecomunicaciones y TIC "Argentina Digital» (27.078/14).

El DNU 267/15 disolvió y unificó las autoridades federales de Servicios de Comunicación Audiovisual (AFSCA) y de Tecnologías de la Información y las Comunicaciones (AFTIC), y eliminó los Consejos Federales de Comunicación Audiovisual y de Tecnologías de las Telecomunicaciones y la Digitalización. Además, creó una Comisión para la elaboración del Proyecto de Ley de Reforma, Actualización y Unificación de las leyes 26.522 y 27.078 antes mencionadas.

Cabe recordar que la LSCA establecía las normas para regular el funcionamiento y la distribución de licencias de los medios radiales y televisivos en el territorio argentino. En este sentido, había determinado límites a la concentración y fijado topes a la cantidad de licencias:

- Un mismo concesionario solo estaba autorizado a tener una licencia de servicio de comunicación audiovisual sobre soporte satelital; hasta 10 señales de radio y de televisión abierta o cable y hasta 24 licencias de radiodifusión por suscripción.

- A ningún operador se le permitía brindar servicios a más de 35\% del total de la población del país o de los abonados, en el caso que corresponda.

- Se impedía que quien manejara un canal de televisión abierta fuera dueño de una empresa de distribución de TV por cable en la misma localidad y viceversa.

- Se prohibía que las compañias telefónicas brindaran servicios de televisión por cable.

- Se fijaba que la duración de las licencias para prestar servicios audiovisuales sería de diez años. Se podrian prorrogar por otros diez, previa realización de audiencias públicas. Quienes hubieran 
obtenido una renovación o una prórroga no estaban autorizados para solicitar una nueva extensión de plazo por ningún título.

- Se reservaba 33\% del espectro radioeléctrico, en radio y televisión, para organizaciones sin fines de lucro, otro 33\% para medios estatales y el último tercio para medios de comunicación con fines comerciales.

Luego de la sanción de la LSCA, el Grupo Clarín impugnó la aplicación de cuatro de sus artículos $(41,45,48$ y 161) mediante el uso de sucesivas medidas cautelares. Los cuestionamientos se centraban en los aspectos que limitaban la acumulación de licencias de televisión por aire y cable, lo que obligaba a la desinversión.

Finalmente, con la asunción del gobierno de Mauricio Macri, se emitió el DNU 267/15, que modificó la LSCA y eliminó varias de sus disposiciones. Esto implicó que el funcionamiento de Clarín y del resto de los medios no tuviera que adecuarse a la norma.

En lo referido a la concentración mediática, el mencionado DNU introdujo los siguientes cambios (Becerra, 2016):

- Elevó de diez a quince la cantidad de licencias de radio y de televisión abierta a nivel nacional, y de tres a cuatro las licencias en el orden local.

- La TV por cable dejó de estar regulada por la LSCA. Con ello, el DNU eliminó el límite para licencias y el porcentaje de dominio de mercado de los cableoperadores.

- Eliminó las reglas que, según la LSCA, impedían la concentración cruzada entre licenciatarios de TV abierta y de TV por cable.

- Eliminó el límite de 35\% de la cobertura de audiencia potencial para emisores de TV abierta y de radio.

- Todos los licenciatarios resultaron beneficiados con la introducción de una "prórroga automática" de cinco años a toda licencia audiovisual y se relajaron las condiciones para que, después, los licenciatarios tramiten una nueva prórroga por otros diez años. 
- Habilitó el cruce para que multimedios audiovisuales puedan brindar servicios de telecomunicaciones y para que, posteriormente, los grupos de telefonía puedan ofrecer televisión por cable.

Con este nuevo marco regulatorio, el 28 de enero de 2016 el Grupo Clarín completó la compra de 100\% de Nextel Argentina, a través de Cablevisión, por un total de U\$S 165 millones. Y en marzo, el fondo de inversión Fintech — socio de Clarín- compró Telecom por U\$S 960 millones. La compra incluyó la empresa de telefonía celular Personal y la de servicios de acceso a Internet Arnet.

El 30 de diciembre de 2016 se sancionó el Decreto 1340, con nuevas disposiciones para el mercado de las telecomunicaciones. El texto establece que se implementan "las normas básicas para alcanzar un mayor grado de convergencia de redes y servicios en condiciones de competencia, promover el despliegue de redes de próxima generación y la penetración del acceso a Internet de banda ancha en todo el territorio nacional" (Art. 1, en línea).

La normativa establece que, a partir del 1 de enero de 2018, las empresas de telefonía fija y móvil (Telefónica, Telecom y Claro) pueden prestar televisión paga por sus propias redes, pero solo en ciudades con más de 80.000 habitantes (Buenos Aires, Córdoba y Rosario); que los grupos de comunicación audiovisual pueden ingresar sin restricciones en el mercado de telefonía fija y móvil —negocio que pretendía Clarín tras la compra de Nextel-; y que los cableoperadores más pequeños (Supercanal y Telecentro) también pueden ofrecer telefonía fija por sus redes.

El decreto prohíbe, en tanto, que las telefónicas y los cableoperadores que ya prestan servicios terrestres puedan ofrecer TV satelital. La finalidad de esta medida es proteger a los cableoperadores pequeños, sobre todo, a las cooperativas y a las PYMES.

También extiende un privilegio por quince años a aquellas empresas de telecomunicaciones que instalen redes de última milla. Este segmento de la red conecta el domicilio del usuario con el resto de la red de telecomunicaciones. Durante ese periodo, otras empresas no podrán emplear el tramo directo al abonado, lo que evita la posibilidad de competencia. 
Además, el decreto atribuye amplias facultades a la autoridad de aplicación y al Ministerio de Comunicaciones para la asignación a demanda de espectro radioeléctrico, el otorgamiento de compensaciones por la retribución de frecuencias, el llamado a concurso para la atribución de nuevas bandas para la prestación de servicios móviles, y el establecimiento de tarifas asimétricas de interconexión y de precios de referencia.

Así las cosas, Clarín podría brindar telefonía móvil, pero pasaría a tener nuevos competidores en el mercado del cable; Telefónica podría ingresar el negocio de la TV paga en Buenos Aires, en Córdoba y en Rosario a partir de 2018; y Direct TV estaría autorizado a ofrecer acceso a Internet.

Esta disposición, sin embargo, no dejó conforme a nadie. Telefónica objetaba que su ingreso a la TV paga en 2018, solo en Buenos Aires, en Rosario y en Córdoba, le otorgaba ventaja a Clarín, que ya detentaba la supremacía en ese mercado. Además, reclamaba por no poder brindar servicios de TV satelital, para lo cual estaba preparada. Clarín, por el contrario, esgrimía que la facturación de TV por cable de Telefónica y de Claro en Latinoamérica multiplicaba por veinte la suya en la Argentina. También argumentaba que el privilegio por quince años de la última milla bloqueaba la posibilidad de competencia.

\section{La fusión Cablevisión-Telecom}

A mediados de 2017, se informó que Cablevisión y Telecom serían los protagonistas de una nueva fusión. Antes de la operación, 60\% de Cablevisión S.A. ${ }^{5}$ estaba en manos de Cablevisión Holding S.A. (compañía controlada por los accionistas del Grupo Clarín, ${ }^{6}$ pero escindida del Grupo), mientras que 40\% restante estaba en posesión de David Martínez, a través de Fintech Media LLC. ${ }^{7}$ A su vez, Fintech Advisory Inc. (controlante de Fintech Media LLC) poseía 100\% de Fintech Telecom LLC. Como Telecom estaba valuada en US\$ 5.000 millones y Cablevisión en US\$ 6.111 millones, se optó por un canje de acciones para concretar la fusión por absorción. En la nueva composición, Fintech tendría 41\% de las acciones y Cablevisión Holding, 33\%. El resto del paquete cotizaría en el mercado. 
De esta manera, Cablevisión-Telecom concentrará a nivel nacional 39\% de la telefonía fija, 33\% de la telefonía móvil, 55\% de las conexiones a Internet de banda ancha y 39\% de TV de paga. El nuevo multimedio tendrá, así, el primer lugar en Internet (Fibertel + Arnet) y en TV paga (Cablevisión), el segundo lugar en telefonía fija y quedará a dos o tres puntos de pasar al primer lugar en telefonía celular (Personal + Nextel). La fusión implicará reducir de tres a dos los operadores más importantes en el acceso a Internet (Fibertel + Arnet, por un lado, y Speedy, por el otro).

Si se toma el indice Hirschman-Herfindahl (HHI), ${ }^{8}$ puede calcularse el grado de concentración en el mercado infocomunicacional de manera sencilla, tanto antes como después de la fusión. Este índice tiene la virtud de ser el más utilizado por las agencias de competencia en el mundo y fue establecido por el Departamento de Justicia de Estados Unidos para evaluar las concentraciones. A partir de este indicador se obtiene un número que varía entre 0 y 10.000, siendo más bajo cuando la distribución de las participaciones es más equitativa y más alto cuando pocas empresas concentran mayores porcentajes de la industria. La escala del índice HHI determina un mercado concentrado (entre 1.800 y 10.000), un mercado de situación intermedia (entre 1.000 y 1.800 ) y un mercado competitivo (entre 0 y 1.000).

En la tabla 2 se muestra la cuota de participación en el mercado (market share) de todas las industrias infocomunicacionales, en los diferentes segmentos, tal y como se encontraban antes de la fusión.

Como se observa, de acuerdo al índice HHI, los niveles de concentración en cada segmento exceden ampliamente el limite de 1.800 , lo que ubica a la estructura del mercado infocomunicacional en la categoría de muy concentrado, incluso antes de la absorción.

En telefonía fija el índice es de 3.625,2; en telefonía móvil, de 3.210,81; en el mercado de acceso a Internet mediante banda ancha, llega al 2.275,5; y en la TV, a 2.598. 


\begin{tabular}{lcccc}
\hline \multicolumn{1}{c}{ Empresa } & $\begin{array}{c}\text { Telefonía } \\
\text { fija }\end{array}$ & $\begin{array}{c}\text { Telefonía } \\
\text { móvil }\end{array}$ & $\begin{array}{c}\text { Banda } \\
\text { Ancha }\end{array}$ & $\begin{array}{c}\text { TV } \\
\text { paga }\end{array}$ \\
\hline Telefónica (España) & $45 \%$ & $33 \%$ & $26 \%$ & - \\
\hline Telecom (David Martínez) & $39 \%$ & $31 \%$ & $24 \%$ & - \\
\hline Clarín & $0,2 \%$ & $2 \%$ & $31 \%$ & $39 \%$ \\
\hline Claro (Telmex) & $3 \%$ & $34 \%$ & - & - \\
\hline Telecentro (Grupo Pierri) & $2 \%$ & - & $4 \%$ & $7 \%$ \\
\hline América (Grupo Vila Manzano) & - & - & $3 \%$ & $7 \%$ \\
\hline $\begin{array}{l}\text { Fecotel, Fecosur y Catel } \\
\text { (Cooperativas telefónicas/eléctricas) }\end{array}$ & $8 \%$ & $0,9 \%$ & $4,5 \%$ & $2 \%$ \\
\hline Red Intercable (Cables pyme) & $1 \%$ & - & $3,5 \%$ & $7 \%$ \\
\hline CCC Tucumán & - & - & - & $1 \%$ \\
\hline $\begin{array}{l}\text { Cablexpress/Gigared/Telered/Antina/ } \\
\text { Phonevision }\end{array}$ & $1 \%$ & - & $2 \%$ & $4 \%$ \\
\hline $\begin{array}{l}\text { Colsecor (Cables cooperativos y } \\
\text { pymes) }\end{array}$ & $0,4 \%$ & - & $1 \%$ & $3 \%$ \\
\hline DirectV (AT\&T Time Warner) & - & - & - & $30 \%$ \\
\hline
\end{tabular}

Tabla 2 | Participación de las industrias infocomunicacionales en el mercado antes de la fusión Fuente: elaboración propia sobre la base de Iglesias (2017)

En la Tabla 3 se muestra cómo quedarian los índices de concentración si se actualizan las participaciones en las cuotas del mercado luego de la fusión entre Cablevisión y Telecom.

\begin{tabular}{|lcccc|}
\hline \multicolumn{1}{c}{ Empresa } & $\begin{array}{c}\text { Telefonía } \\
\text { fija }\end{array}$ & $\begin{array}{c}\text { Telefonía } \\
\text { móvil }\end{array}$ & $\begin{array}{c}\text { Banda } \\
\text { Ancha }\end{array}$ & $\begin{array}{c}\text { TV } \\
\text { paga }\end{array}$ \\
\hline Telefónica (España) & $45 \%$ & $33 \%$ & $26 \%$ & - \\
\hline Telecom-Cablevisión & $39,2 \%$ & $33 \%$ & $55 \%$ & $39 \%$ \\
\hline Claro (Telmex) & $3 \%$ & $34 \%$ & - & - \\
\hline Telecentro (Grupo Pierri) & $2 \%$ & - & $4 \%$ & $7 \%$ \\
\hline América (Grupo Vila Manzano) & - & - & $3 \%$ & $7 \%$ \\
\hline $\begin{array}{l}\text { Fecotel, Fecosur y Catel } \\
\text { (Cooperativas telefónicas/eléctricas) }\end{array}$ & $8 \%$ & $0,9 \%$ & $4,5 \%$ & $2 \%$ \\
\hline Red Intercable (Cables pyme) & $1 \%$ & - & $3,5 \%$ & $7 \%$ \\
\hline CCC Tucumán & $1 \%$ & - & - & $1 \%$ \\
\hline $\begin{array}{l}\text { Cablexpress/Gigared/Telered/Antina/ } \\
\text { Phonevision }\end{array}$ & $0,4 \%$ & - & $2 \%$ & $4 \%$ \\
\hline $\begin{array}{l}\text { Colsecor (Cables cooperativos y } \\
\text { pymes) }\end{array}$ & - & - & - & $30 \%$ \\
\hline DirecTV (AT\&T Time Warner) & $\mathbf{3 6 4 0 , 8}$ & $\mathbf{3 3 3 4 , 8 1}$ & $\mathbf{3 7 6 3 , 5}$ & $\mathbf{2 5 9 8}$ \\
\hline
\end{tabular}

Tabla 3 | Participación de las industrias infocomunicacionales en el mercado luego de la fusión Fuente: elaboración propia sobre la base de Iglesias (2017) 
Como es lógico, el nivel de concentración se incrementa en casi todos los segmentos: pasa de 3.625,2 a 3.640,8 en telefonía fija; de $3.210,81$ a $3.334,81$ en telefonía móvil; y de 2.275,5 a 3.763,5 en el acceso a Internet. El mercado de la TV por cable, por su parte, no registra variación.

El monopolio de las empresas fusionadas es más elevado en regiones de mayor poder adquisitivo y concentración demográfica. Sobre todo, en el centro y en el norte del país, donde Telecom mantiene su área de influencia luego de la privatización de Entel, tal como se detalló anteriormente. En al menos 130 localidades, donde había dos compañias para competir en la oferta de prestaciones del cuádruple play ahora habrá solo una.

El 21 de diciembre de 2017, el directorio del ENaCom -mediante la Resolución 5644-, aprobó administrativamente la transferencia de licencias de Cablevisión S.A (subsidiaria de $\mathrm{CVH}$ ) a Telecom, en el marco del proceso de fusión entre ambas empresas. Como condición para autorizar la operación, se determinó que como ambas compañías sumadas superan el límite de espectro radioeléctrico establecido por la regulación vigente (140 $\mathrm{MHz}$ para operadores móviles), se deberá devolver el excedente en un plazo de dos años. La resolución también regula el precio minorista del servicio de banda ancha fija en aquellas localidades en las que las empresas fusionadas superen $80 \%$ de los abonados. ${ }^{9}$ En estos casos, el precio del servicio no puede superar la menor tarifa que cobra el conglomerado en la Ciudad Autónoma de Buenos Aires (CABA). Finalmente, se obliga a compartir la infraestructura soporte en dichas ciudades en condiciones no discriminatorias a otros prestadores. Estas imposiciones de competencia se extenderán durante dos años y podrán prorrogarse a discreción del ente regulador.

Simultáneamente, a través de la Resolución 5641/17, el ENaCom adelantó el plazo en el que autorizaba a las empresas telefónicas a brindar el servicio de TV por cable en todo el territorio nacional, y no solo en Buenos Aires, Córdoba y Rosario, tal como había establecido el decreto 1340 en 2016. La única limitación al respecto son las localidades con menos de 80 mil habitantes y aquellas en las que, superando ese número, existan únicamente operadores cooperativos o PYMES que ofrezcan ese servicio. Además, ninguna telefónica podrá prestar TV por cable en localidades donde operen empresas que no superen $10 \%$ de abonados a nivel nacional, pero solo hasta el 1 de enero de 2019. 
La sugestiva decisión de anticipar el plazo que permite el ingreso de las telefónicas en el mercado de la TV por cable parecería estar destinada a facilitar la fusión entre Cablevisión y Telecom. En efecto, sin esta disposición, la empresa fusionada se vería en dificultades para desplegar las operaciones de Cablevisión en el mercado de la TV paga con presencia en todo el territorio nacional. La única limitación que dispone la normativa, tanto para Telecom como para sus competidores (Telefónica y Claro), es integrar servicios en un mismo paquete fuera de los grandes centros urbanos hasta 2019.

Actualmente, la operación de fusión espera la autorización de la Comisión Nacional de Defensa de la Competencia, pero la aprobación se da, prácticamente, por descontada.

Cabe aclarar que la singularidad de la fusión reside en que no se trata únicamente de una cuestión de conectividad sino también de producción de contenidos. Justamente, el Grupo Clarín ${ }^{10}$ es el mayor editor de diarios (Clarin, Olé, La Razón, La Voz del Interior y Los Andes); propietario de radios líderes en AM (Radio Mitre) y en FM (La 100), y de una de las cadenas de televisión abiertas más importantes (El Trece). Además, es accionista mayoritario en Papel Prensa S.A. y posee varias señales de televisión paga (Becerra, 2017). A la totalidad de la cadena productiva de contenidos en comunicación, debe sumarse ahora la posición dominante en las redes de transporte, de distribución y de comercialización de datos a escala nacional.

\section{Conclusiones}

Como se desarrolló en este texto, la digitalización de las telecomunicaciones permite la integración de las distintas redes de datos para transportar servicios y señales similares. Simultáneamente, ofrece la posibilidad de recibir diversos servicios a través de un mismo dispositivo. De este modo, la convergencia tecnológica implica nuevas formas de producción, de circulación y de consumo de bienes culturales.

La otra cara de este fenómeno es la convergencia de los mercados, que implica, necesariamente, un desafio para las políticas de comunicación, dado que la importancia de las regulaciones no radica simplemente en ordenar el funcionamiento económico de las industrias del sector. 
La concentración mediática y el interés público no pueden ser disociados en el análisis de la conformación de los sistemas de medios de comunicación y de las industrias que producen y que distribuyen información y entretenimiento a escala masiva. En el orden democrático, la función de las industrias de la comunicación es medular para estructurar espacios públicos deliberativos (Sorj, 2012) que permitan el intercambio de informaciones, de ideas y de opiniones diversas. La concentración del sector no puede escindirse del análisis sobre las condiciones en las que los individuos y los grupos socializan (Becerra, 2015).

La dimensión política de la concentración mediática no se explica solamente por el modo en que la cultura, las ideas y la información circulan de forma masiva en la sociedad, sino que, además, se apoya en la doctrina internacional sobre derechos humanos que privilegia el derecho a la expresión, a la libre información y a la cultura (Loreti, 2013).

La breve reconstrucción histórica realizada a lo largo de las páginas precedentes muestra que la concentración del mercado infocomunicacional tiende a un constante incremento. La fusión entre Cablevisión y Telecom es una prueba evidente de este fenómeno. La dinámica monopólica del capitalismo junto con la convergencia tecnológica, por un lado, y las políticas de comunicación llevadas a cabo desde 2015, por el otro, provocan que la oferta de prestadores sea cada vez más reducida. Si bien las consecuencias políticas ligadas al interés público exceden las pretensiones de este artículo, resulta claro que la concentración mediática es absolutamente contraria a libertad de expresión y de información, y tiene efectos nocivos sobre el orden democrático (Sorj, 2012; Gómez, 2018).

Sin embargo, todas las políticas regulatorias parecerían dirigirse en ese sentido. El 1 de marzo de 2018, el Gobierno Nacional anunció que no dictaría un nuevo decreto para habilitar a Telefónica y a Claro a brindar televisión satelital, tal como estaba previsto. Si bien es cierto que se permite a ambas empresas el ingreso al mercado de la TV paga, existe un inconveniente: las redes de estas compañias no pueden transmitir televisión por cable debido a que la mayor parte de su tendido se compone de cobre, un material no apto para estas señales como la fibra óptica. Por eso, desde 2017 las telefónicas mantuvieron negociaciones con el Gobierno para que se las habilite a dar televisión satelital. 
Por su parte, la presión del Grupo Clarín se dirige a que no se permita el ingreso de Telefónica y de Claro al mercado televisivo por satélite. $\mathrm{Su}$ argumento es que estas empresas no invirtieron, como Cablevisión, en el tendido de fibra óptica, cosa que tampoco harían, si se les permitiera el uso de satélites. Al fusionarse Telecom con Cablevisión se crea una empresa con posibilidad de dar cuádruple play de inmediato, sin una competencia real en el país, al menos, por el momento. La autorización de brindar TV satelital era una promesa para equilibrar el mercado luego de la fusión en favor de las telefónicas perjudicadas.

Presumiblemente, con esa intención, el 5 de abril de 2018, el oficialismo presentó el proyecto de Ley de Fomento y Despliegue de Infraestructura y Competencia de Tecnologías de la Información y las Comunicaciones (TIC). Al momento de redacción de este artículo, dicho proyecto se encuentra en debate en las comisiones de Sistemas, Medios de Comunicación y Libertad de Expresión, y de Educación y Cultura del Senado de la Nación.

El texto apunta a regular cuatro cuestiones:

- Establecer la obligatoriedad de compartir infraestructura pasiva entre los licenciatarios de servicios TIC mediante convenios en forma "objetiva, transparente, no discriminatoria y proporcionada", con el fin de favorecer el despliegue de redes.

- Licitar, entre privados, la reserva preferente de 90 megas de espectro, actualmente en manos de ARSAT, para destinarlo a operadores móviles de telefonía celular.

- Permitir, a los prestadores de telecomunicaciones, brindar servicios de radiodifusión por suscripción mediante vínculo satelital a partir del 1 de enero de 2019 en localidades de menos de 80 mil habitantes. Este plazo tendría el fin de proteger a los operadores cooperativos y PYMES del interior del país.

- Establecer la comercialización de señales o de programas audiovisuales en condiciones "transparentes, equitativas y no discriminatorias", para garantizar la competencia leal y efectiva entre los licenciatarios TIC. 
En principio, de no introducirse modificaciones en el proyecto, la normativa resultante parecería estar hecha a medida de las grandes empresas de telecomunicaciones y en claro detrimento de las cooperativas y las PYMES.

En primer lugar, el texto tiende a soslayar las abismales asimetrias geográficas y económicas que existen en el mercado nacional. Los pequeños cableoperadores, proveedores de Internet y de telefonía móvil del interior del país, simplemente no se encuentran en posición de competir en igualdad de condiciones contra empresas multinacionales que operan a nivel regional. $\mathrm{Si}$, además, se tiene en cuenta que estas cooperativas y PYMES producen contenido local y generan puestos de trabajo, entonces, las consecuencias de no tomar medidas asimétricas para asegurar su viabilidad económica pueden ser desastrosas.

En segundo lugar, el plazo proteccionista de un año que limita el ingreso de las telefónicas a las localidades pequeñas, parece ser a todas luces muy exiguo. Además, si no está acompañado con políticas de incentivos para favorecer, por ejemplo, la compra de equipamiento tecnológico o el acceso a la licitación de espectro en condiciones de igualdad, la competencia de los pequeños operadores resultará imposible. La obligación de incorporar el contenido audiovisual generado por las señales locales a las grillas de los operadores satelitales es otra medida imprescindible para impedir la desaparición de las identidades culturales diversas.

Ante este panorama, el futuro de los distintos actores dentro del mercado infocomunicacional parece incierto y dependerá, en gran medida, de la capacidad para imponer sus demandas en la instancia del debate legislativo que aún está por resolverse.

\section{Referencias}

Becerra, M. (2015). De la concentración a la convergencia. Politica de medios en Argentina y América Latina. Buenos Aires, Argentina: Paidós. 
Becerra, M. (2016). "Restauración" [entrada de blog]. Quipu. Políticas y tecnologías de comunicación. Recuperado de https://martinbecerra.wordpress.com/2016/01/14/restauracion

Becerra, M. (2017). "Concentración extrema: Cablevisión + Telecom" [entrada de blog]. Recuperado de https: / / martinbecerra.wordpress.com/2017/07/01/concentra cion-extrema-cablevision-telecom/\#more-5548

Becerra, M. y Mastrini, G. (2017). La concentración infocomunicacional en América Latina 2000-2015. Nuevos medios y tecnologías, menos actores. Bernal, Argentina: Universidad Nacional de Quilmes.

Bolaño, C. y Mastrini, G. (2002). Economía Política de la Comunicación: un aporte marxista a la constitución del campo comunicacional. Oficios Terrestres, (11-12), 45-55. Recuperado de http://sedici.unlp.edu.ar/handle/10915/46764

Cammisa, M. y Zalazar, G. (2015). "Análisis del status de los servicios de telecomunicaciones y proyección de la inversión para el período 2016-2025». Ciudad Autónoma de Buenos Aires, Argentina: Cámara Argentina de la Construcción. Recuperado de http: / / www.camarco.org.ar/File/GetPublicFile?id=3548

Castañeda Véliz, C. (2007). "Indicadores de concentración. Una revisión del marco conceptual y la experiencia internacional». Documento de Trabajo $N^{\circ}$ 002-2007. Lima, Perú: Organismo Supervisor de Inversión Privada en Telecomunicaciones (OSIPTEL).

Dantas, M. (Coord.) (2013). Avances en los procesos de democratización de la comunicación en América Latina. Buenos Aires, Argentina: Consejo Latinoamericano de Ciencias Sociales (CLACSO). 
Garnham, N. (1990). Capitalism and Communication. Londres, Inglaterra: Sage Publications.

Gómez, G. (Coord.) (2018). ¿Quién es el guardián del perro guardián? Historias de cómo la concentración de medios afecta el periodismo y la libertad de expresión. Observatorio Latinoamericano de Regulación, Medios y Convergencia (OBSERVCOM).

Iglesias, R. (2017). La convergencia digital en América. Un viaje por las empresas, los mercados y la regulación de las comunicaciones. Colección Cuadernos Convercom. Recuperado de http:/ / convercom.org/site/wpcontent/uploads/2017/10/La-convergencia-digital-enAme\%CC\%81rica-completo-y-final.pdf

Jenkins, H. (2006). Convergence Culture: Where Old and New Media Collide. New York, Estados Unidos: New York University Press.

Kerlinger, F. y Lee, H. (2002). Investigación del comportamiento. Ciudad de México, México: Mcgraw-Hill / Interamericana Editores.

Lessig, L. (1998). Las leyes del ciberespacio. Cuadernos Ciberespacio y Sociedad, (3).

Loreti, D. (2013). "El caso argentino. Acerca de la nueva Ley de Servicios de Comunicación Audiovisual N. o 26.522". Revista Zigurat, (7), 8-13.

Meikle, G. y Young, S. (2011). Media Convergence: Networked Digital Media in Everyday Life. Basingstoke, Inglaterra: Palgrave Macmillan. 
Miguel de Bustos, J. C. (1993). Los grupos multimedia: estructuras y estrategias en los medios europeos. Barcelona, España: Bosch.

Miller, V. (2011). Understanding Digital Culture. Londres, Inglaterra: Thousand Oaks Sage.

Mosco, V. (2009). The Political Economy of Communication. Londres, Inglaterra: Sage Publications.

Peil, C. y Sparviero, S. (2017). "Media Convergence Meets Deconvergence». En S. Sparviero y otros (Eds.). Media Convergence and Deconvergence, Global Transformations in Media and Communication Research. Londres, Inglaterra: Palgrave Macmillan.

Sánchez Ruiz, E. (1991). Medios de difusión y sociedad. Ciudad de México, México: Ediciones Universidad de Guadalajara.

Sel, S. (Comp.) (2010). Politicas de comunicación en el capitalismo contemporáneo. Buenos Aires, Argentina: Consejo Latinoamericano de Ciencias Sociales (CLACSO).

Sorj, B. (Coord.) (2012). Democracia y medios de comunicación. Más allá del estado y el mercado. Buenos Aires, Argentina: Catálogos.

Williams, R. [1974] (1997). La politica del modernismo: contra los nuevos conformistas. Buenos Aires, Argentina: Manantial. 
Cablevisión Holding S.A. Memoria y estados financieros consolidados (del 1 de mayo al 31 de diciembre de 2017). Recuperado de

https: / / www.cablevisionholding.com/files / Estados-

Financieros/CVH\%20-\%20EEFF\%20-\%2012-2017\%20\%20AIF\%20-\%20desprotegido.pdf

Cablevisión Holding S.A. (2018). Investor Presentation. Recuperado de

https:/ / www.cablevisionholding.com/files / Presentaciones / CVH \%20-\%20March\%202018_as\%20of\%20June\%202018_vf_v2.pdf

Decreto 1340 (2 de enero de 2017). Ministerio de Comunicaciones. Normas Básicas - Implementación. Recuperado de http:// servicios.infoleg.gob.ar/infolegInternet/verNorma.do?id= 270115

Decreto de Necesidad y Urgencia 267 (29 de diciembre de 2015). Ente Nacional de Comunicaciones. Creación Modificación Leyes $\mathrm{N}^{\circ} 26.522$ y N²7.078. Recuperado de http:/ / servicios.infoleg.gob.ar/infolegInternet/verNorma.do?id $=257461$

ENACOM (Ente Nacional de Comunicaciones) (2017). Indicadores de Mercado TIC y Audiovisual, Argentina (2017). Recuperado de https://indicadores.enacom.gob.ar/Default.aspx

Ley 25.156 (20 de septiembre de 1999). Defensa de la Competencia. Nuevo Régimen Legal. Recuperado de http:/ / servicios.infoleg.gob.ar/infolegInternet/verNorma.do?id $=60016$ 
Ley 26.305 (19 de diciembre de 2007). Convención sobre la Protección y Promoción de la Diversidad de las Expresiones Culturales. Recuperado de

http://servicios.infoleg.gob.ar/infolegInternet/verNorma.do?id $=135906$

Ley 26.522 (10 de octubre de 2009). Servicios de Comunicación Audiovisual. Regulación. Recuperado de http:/ / servicios.infoleg.gob.ar/infolegInternet/verNorma.do?id $=158649$

Ley 27.078 (19 de diciembre de 2014). Argentina digital. Tecnologías de la Información y las Comunicaciones. Recuperado de http://servicios.infoleg.gob.ar/infolegInternet/verNorma.do?id $=239771$

Proyecto Ley de Fomento de Despliegue de Infraestructura y Competencia TICS (5 de abril de 2018). Recuperado de https:/ /www.parlamentario.com/db/000/000604_proyecto_le y_telecomunicaciones.pdf

Resolución 5641 (22 de diciembre de 2017). Ente Nacional de Comunicaciones. Plazo - Prórroga. Recuperado de http:/ / servicios.infoleg.gob.ar/infolegInternet/verNorma.do?id $=305089$

Resolución 5644 (22 de diciembre de 2017). Ente Nacional de Comunicaciones. Cablevisión Sociedad Anónima. Recuperado de http:/ / servicios.infoleg.gob.ar/infolegInternet/verNorma.do;jsessi onid=18EB35C9A92FA9A897C8898E51CCF0D4?id=305088

Telecom Argentina S.A. Estados financieros contables al 30 de septiembre de 2017. Recuperado de https://www.bolsar.com/Vistas/sociedades/FichaTecnicaSoci edadesDetalle.aspx?EmiID $=208$ 
1 Estados financieros contables de Telecom Argentina S.A. al 30 de septiembre de 2017.

2 Memoria y Estados Financieros Consolidados de Cablevisión Holding S.A. del $1^{\circ}$ de mayo al 31 de diciembre de 2017.

3 La noción "infocomunicación" se utiliza de modo analítico para aludir en un mismo concepto a todas las industrias y las actividades de información y de comunicación como la industria gráfica - libros, revistas y diarios-, audiovisual -televisión, cine y radio- y de telecomunicaciones e informática (Becerra, 2015).

4 Para un estudio detallado de las inversiones en el sector infocomunicacional, ver el trabajo de Cammisa \& Zalazar (2015).

5 Al momento de la fusión, Cablevisión participaba en las siguientes sociedades constituidas de conformidad con las leyes de la República Argentina: Pem S.A. (99,9\%), una sociedad inversora; Cable Imagen S.R.L. (100\%), una emisora de televisión por cable; Ver TV S.A. (49\%), una emisora de televisión por cable; Teledifusora San Miguel Arcángel S.A. (49,1\%), una emisora de televisión por cable; La Capital Cable S.A. (49\%), cuya actividad principal es la televisión por circuito cerrado; y Última Milla S.A. (95\%), dedicada al tendido de redes de comunicación (el restante $5 \%$ está en poder de Pem S.A.).

Cablevisión posee participación directa e indirecta en las siguientes sociedades constituidas en el extranjero: en la emisora de televisión por cable Televisión Dirigida S.A. (Paraguay) y en la sociedad inversora Adesol S.A. (Uruguay); en ambos casos, con 100\% de las acciones. A su vez, Adesol S.A. tiene participación en las siguientes empresas constituidas en Uruguay dedicadas a la venta y a la instalación de servicios de televisión por cable para abonados y de servicios de televisión codificada: Audomar S.A. $(0,1 \%)$ y Bersabel S.A. $(1,2 \%)$; en las siguientes sociedades dedicadas a la venta y a la instalación de servicios de televisión por cable: Dolfycor S.A. (0,5\%), Reiford S.A. $(0,3 \%)$ y Space Energy Tech S.A. (0,47\%); y en las siguientes compañías dedicadas a la venta y a la instalación de servicios de televisión codificada para abonados: Tracel S.A. (2,5\%), Visión Satelital S.A. $(0,44 \%)$ y Telemas S.A. (100\%); esta última, dedicada a la administración de empresas relacionadas con las actividades de televisión por cable para abonados. 
Por otra parte, Cablevisión participa indirectamente a través de Pem S.A. en las siguientes sociedades constituidas en la Argentina dedicadas a la emisión de televisión por cable: CV Berazategui S.A. (70\%), AVC Continente Audiovisual S.A. (40\%) y Televisora Privada del Oeste S.A. (47\%). También participa indirectamente en Otamendi Cable Color S.A., una emisora de televisión por cable de la cual La Capital Cable S.A. posee $50 \%$ de las acciones, y en First TV S.A. de la cual Ver TV S.A. tiene 95\% de las acciones.

A su vez, Cablevisión era titular de $100 \%$ de las cuotas sociales de Nextel Communications Argentina S.R.L., sociedad a la cual absorbió por fusión junto con las controladas por Nextel: WX Telecomunication S.A.U., Greenmax Telecomunications S.A.U., Gridley Investments S.A., Trixco S.A., Fibercomm S.A., Netizen S.A., Eritown Corporation S.A., Skyonline de Argentina S.A., Infotel Argentina S.A., Nextwave Argentina S.A. y Callbi S.A., de acuerdo a lo decidido en asamblea de accionistas del 31 de marzo de 2017 (Cablevision Holding, 2018, en línea).

6 Los accionistas de Grupo Clarin son: New York Trust (Ernestina Laura Herrera de Noble), HHM Media New York Trust (Héctor Horacio Magnetto), The LRP New York Trust (Lucio Rafael Pagliaro), GC Dominio S.A y José Antonio Aranda (Cablevision Holding, 2018, en linea).

7 En rigor, FINTECH MEDIA LLC tenía participación en CABLEVISIÓN S.A. a través de VLG ARGENTINA LLC en copropiedad con CABLEVISIÓN HOLDING S.A. VLG ARGENTINA llevó a cabo un proceso de reorganización societaria en virtud del cual escindió proporcionalmente a las respectivas tenencias de sus accionistas FINTECH MEDIA LLC $(28,45 \%)$ y CABLEVISIÓN HOLDING $(71,55 \%)$ parte de su patrimonio para la constitución de una nueva sociedad a denominarse "VLG ARGENTINA ESCINDIDA LLC", todo ello con efecto a la fecha efectiva de fusión. Como consecuencia de ese proceso, CABLEVISIÓN HOLDING resultó ser titular del 100\% de VLG ARGENTINA que fue la continuadora de la titularidad de CABLEVISIÓN y FINTECH MEDIA LLC resultó ser titular del 100\% de VLG ARGENTINA ESCINDIDA LLC. Asimismo, FINTECH TELECOM, FINTECH MEDIA y VLG ARGENTINA ESCINDIDA LLC, llevaron a cabo un proceso de reorganización societaria interna por la cual FINTECH MEDIA y VLG ARGENTINA ESCINDIDA LLC se fusionaron con FINTECH TELECOM, con efecto a la fecha efectiva de fusión (Cablevision Holding, 2018, en línea). 
8 En rigor, el índice se calcula elevando al cuadrado la cuota de mercado que cada empresa posee y sumando esas cantidades. Un índice elevado expresa un mercado muy concentrado y poco competitivo en una escala entre 0 (competencia perfecta) y 10.000 (monopolio absoluto). Para más detalles, ver el trabajo de Castañeda Veliz (2007).

9 De acuerdo al Anexo I de la Resolución 5644/2017, las localidades en las cuales la empresa fusionada contará con más de $80 \%$ de los abonados de Internet Fija son: Baradero, San Nicolás de los Arroyos, San Pedro y Villa Ramallo (provincia de Buenos Aires); Córdoba Capital, Bell Ville, Cosquín, Jesús María, La Calera, La Falda, Laboulaye, Leones, Mendiolaza, Río Ceballos, Río Cuarto, Saldán, San Francisco, Unquillo, Villa Carlos Paz, Villa María y Villa Nueva (provincia de Córdoba); Concepción del Uruguay, Gualeguay, Gualeguaychú y San Benito (provincia de Entre Ríos); Arroyo Seco, Cañada de Gómez, Casilda, Esperanza, Firmat, Rafaela, San Carlos Centro, San Lorenzo, Santa Fe y Villa Constitución (provincia de Santa Fe); Formosa capital (provincia de Formosa); Cerrillos (provincia de Salta).

10 Grupo Clarin controla, directa e indirectamente, las siguientes empresas con actividad en Argentina: GC MINOR S.A. (95\%), GCSA INVESTMENTS S.A.U. (100\%) y GC GESTIÓN COMPARTIDA S.A. (97,5\%). ARTES GRÁFICOS RIOPLATENSE S.A. (22,2\%). ARTE GRÁFICO EDITORIAL ARGENTINO S.A. (97\%). AGEA, participa en: i) TINTA FRESCA EDICIONES S.A. (95\%); ii) FERIAS Y EXPOSICIONES S.A. (95\%), que a su vez tiene el 50\% de EXPONENCIAR S.A; iii) UNIR S.A. (93,4\%); iv) GRATUITOS DEL AIRE S.A. (50\%); v) DIARIOS Y NOTICIAS S.A. (29,6\%); vi) OPORTUNIDADES S.A. (95\%). Esta sociedad, a su vez, participa con 95\% en MAS LOGÍSTICA S.A. y en DISTRITO B S.A., v) COMPAÑIIA INVERSORA EN MEDIOS DE COMUNICACIÓN S.A. $(67,7 \%)$. Asimismo, AGEA también participa en PAPEL PRENSA S.A.C.I.F y de M. (36,99\%), COMPAÑÍA INVERSORA EN MEDIOS DE COMUNICACIÓN S.A. (CIMECO) (12\%); ii) LA NACIÓN (22,48\%); iii) ESTADO NACIONAL (27,46\%); iv) otros inversores (1,052\%); v) TELAM $(0,67 \%)$. CIMECO, en tanto, participa en las siguientes sociedades: i) COMERCIALIZADORA DE MEDIOS DEL INTERIOR S.A. (97\%); ii) VÍA ROSARIO S.A. (95\%); iii) DIARIO LOS ANDES HNOS. CALLE S.A. (80\%); iv) LA VOZ DEL INTERIOR S.A (81.33\%). DIARIO LOS ANDES HNOS. CALLE S.A. tiene participación en: a) CUYO TELEVISIÓN S.A. (9\%); b) ANDES SATELITAL S.A. (99,3\%); c) INTERDIARIOS S.A. (98\%); d) DIARIOS Y NOTICIAS S.A. (3,9\%). LA VOZ DEL INTERIOR S.A. controla con 100\% de las acciones a CONTENIDOS MEDITERRÁNEOS S.A. COMPAÑíA DE MEDIOS DIGITALES 
S.A. (98\%), tiene participación en las siguientes sociedades: i) INTERWA S.A. (100\%); ii) CLAWI S.A. (51\%); iii) FYNBAR S.A. (100\%), iv) ELECTRO PUNTO NET S.A. (65,56\%), v) QB9 S.A. (95\%); vi) TECNOLOGÍA DIGITAL S.A. (98\%). ARTE RADIO TELEVISIVO ARGENTINO S.A. (96,9\%) participa en las siguientes sociedades: i) CARRIERSAT S.A. (93,97\%); ii) PATAGONIK FILM GROUP S.A. (33,33\%), que a su vez controla con el 99\% de las acciones a PATAGONIK ANIMATION GROUP S.A.; iii) POLKA PRODUCCIONES S.A.) (55\%); iv) SB PRODUCCIONES S.A. (55\%); v) TELECOR S.A.CI. (85,19\%); vi) BARILOCHE TV S.A. (99,90\%); vii) CANAL RURAL SATELITAL S.A. (40\%); viii) TELEDIFUSORA BAHIENSE S.A. (100\%); ix) ARTECORP S.A. (50\%); $x$ ) GOTILAND S.A. (50\%); xi) RPA MEDIA PLACE S.A. (19\%); xii) SI MUSICA S.A. (95\%). BARILOCHE TV S.A. participa con el 94\% de las acciones en BARILOCHE 2000 S.A., dedicada a prestar servicios periodísticos y publicitarios. INVERSORA DE EVENTOS S.A. (96\%), tiene participación en: i) TELE RED IMAGEN S.A. (50\%); ii) TELEVISIÓN SATELITAL CODIFICADA S.A. (50\%); iii) CARBURANDO S.A. (95,55\%); iv) AUTO SPORTS S.A. (95,76\%). RADIO MITRE S.A. (95\%) (Cablevision Holding, 2018, en línea). 